\title{
SPATIAL MEMORY PERFOMANCE OF WISTAR RATS EXPOSED TO MOBILE PHONE
}

\author{
Sareesh Naduvil Narayanan, ${ }^{\mathrm{I}}$ Raju Suresh Kumar, ${ }^{\mathrm{I}}$ Bhagath Kumar Potu, ${ }^{\mathrm{II}}$ \\ Satheesha Nayak, ${ }^{\mathrm{III}}$ Maneesh Mailankot ${ }^{\mathrm{IV}}$
}

doi: $10.1590 / \mathbf{S 1 8 0 7 - 5 9 3 2 2 0 0 9 0 0 0 3 0 0 0 1 4}$

Sareesh N N, Kumar RS, Potu BK, Nayak S, Maneesh M. Spatial memory perfomance of wistar rats exposed to mobile phone. Clinics. 2009;64(3):231-4.

INTRODUCTION: With the tremendous increase in number of mobile phone users world wide, the possible risks of this technology have become a serious concern.

OBJECTIVE: We tested the effects of mobile phone exposure on spatial memory performance.

MATERIALS AND METHODS: Male Wistar rats (10-12 weeks old) were exposed to 50 missed calls/day for 4 weeks from a GSM (900/1800MHz) mobile phone in vibratory mode (no ring tone). After the experimental period, the animals were tested for spatial memory performance using the Morris water maze test.

RESULTS: Both phone exposed and control animals showed a significant decrease in escape time with training. Phone exposed animals had significantly ( $\sim 3$ times) higher mean latency to reach the target quadrant and spent significantly ( 2 times) less time in the target quadrant than age- and sex-matched controls.

CONCLUSION: Mobile phone exposure affected the acquisition of learned responses in Wistar rats. This in turn points to the poor spatial navigation and the object place configurations of the phone-exposed animals.

KEYWORDS: Mobile phone; Rats; Memory; Learning; Water maze.

\section{INTRODUCTION}

The recent enormous increase in the use of wireless mobile devices has motivated a large body of research, both epidemiological and experimental. This voluntary exposure to microwaves has been called the largest human biological experiment ever performed. The possible risks of these microwaves are of growing concern in our society. Exposure to an electromagnetic field (EMF) at $1.8 \mathrm{GHz}$ increases the

Departments of 'Physiology, ${ }^{\mathrm{II}}$ Anatomy, and ${ }^{\mathrm{IV}}$ Biochemistry, Melaka Manipal Medical College - Manipal, India.

"Department of Anatomy, Kasturba Medical College - Manipal, India. Tel.: 918202922637

Email: manu_only@hotmail.com

Received for publication on October 22, 2008

Accepted for publication on November 10, 2008 permeability of the blood-brain barrier to sucrose ${ }^{1}$. The combined exposure to radiofrequency (RF) EMFs and pulsed and static magnetic fields results in significant pinocytotic transport of albumin from the capillaries into the brain ${ }^{2}$. Our recent study (Kumar et al., submitted) has shown that mobile phone exposure in Wistar rats results in high anxiety, demonstrated by deficient open arm exploration on an elevated plus-maze apparatus and by a fear induced increase in parasympathetic activity.

In the present work we studied the spatial memory performance of Wistar rats exposed to mobile phones using the Morris water maze (MWM), the bench mark test for spatial memory performance. Because teenagers are the heaviest users of wireless technology and are of special concern, we exposed 8-10 weeks old Wistar rats, which are developmentally comparable to human teenagers, to EMFs from a GSM mobile phone. 


\section{MATERIALS AND METHODS}

Animals: Male Albino rats of the Wistar strain, 10-12 weeks of age, were housed in plastic cages of dimensions $14 " \times 9 " \times 8$ " (three rats in each cage) inside a well-ventilated room at $22 \pm 2^{\circ} \mathrm{C}$ with a 12 -hr L:D cycle. All animals were provided free access to standard diet and water. The Animal Ethics Committee, Manipal University, Manipal approved all the procedures used. The animals were divided into two groups of six rats each; group I was the control, and group II was exposed to 50 missed calls (separated by $15 \mathrm{sec}$ intervals) per day for 4 weeks, by keeping a GSM $(900 / 1800 \mathrm{MHz})$ mobile phone in vibratory mode (no ring tone) within the cage. Each missed call lasted $1 \mathrm{~min}$. The animals were free to move in the cage and the phone was kept in a 4 " $\times 2$ " $\times 1$ " wood-bottom cage to avoid animal contact with hardware. After the experimental period, all animals were tested for spatial memory performance using the Morris Water Maze (MWM).

Morris water maze: The experiment was carried out in a circular, galvanized steel maze $(1.83 \mathrm{~m}$ in diameter and $75 \mathrm{~cm}$ in depth), which was filled with water $\left(22 \pm 2{ }^{\circ} \mathrm{C}\right)$ to a depth of $50 \mathrm{~cm}$ and rendered opaque by the addition of a non-toxic, water soluble dye. The maze was located in a large and quiet test room, surrounded by many visual cues external to the maze (e.g. the experimenter, celing fan, lights, racks, etc.), which were visible from within the pool and could be used by the rats for spatial location. The locations of the cues were unchanged throughout the period of testing. A video camera was fixed to the ceiling over the centre of the maze to record and monitor the movements of the animals. The pool was divided into four equal quadrants. In one of the quadrants, the target quadrant, a platform $(1.0 \mathrm{~cm}$ below water surface; 4 "x 4 " size) was submerged in a fixed central position. The position of the platform was kept constant throughout the acquisition trials. The rats were trained in the maze over seven sessions on 4 consecutive days, two sessions on each day except on the first day, when only one session was given. In the swimming trials, an individual rat was released gently into the water facing the wall of the maze in each quadrant except in the case of the quadrant that contained the hidden platform, in which case the rat faced an extra-maze cue. The trials were always initiated from different positions in the tank. The rat swam and learned how to find the hidden platform within 180 seconds. After finding the submerged platform, the rat was allowed to rest on the platform for 15 seconds. During the inter-trial intervals, the animals were kept in a dry cage for 60 seconds. The escape latency represents the time until the rat reached the platform in each trial which is considered a measure of the acquisition of spatial navigation abilities. We recorded this value in seconds, and the mean daily escape latency was calculated thereafter. If the animal were unable to escape to the platform within 60 seconds unassisted, their escape latency was recorded as 60 seconds. To assess their spatial memory, the platform was removed from the maze. The animals were tested for retention of spatial memory 24 hours after the final trail. In this trial, lasting for $30 \mathrm{sec}$, each rat was placed into the water as in the training trails. Time taken to reach the target quadrant and time spent in the target quadrant were recorded.

Statistical analysis was performed by using Students ' $t$ ' test and the significance level was set at $\mathrm{p}<0.05$.

\section{RESULTS}

During the training sessions, both groups showed significant trial effects (i.e. a significant decrease in escape time with training) (Figure 1). There was also a notable difference between the two groups with respect to the escape time during the training trials. Both groups of animals improved their water maze acquisition performance i.e. decreased the latency to find the platform from the first to the last day of training (day 4). During the probe trial, with the platform removed, rats exposed to mobile phones were unable to remember the location of the platform. The mean latency to reach the target quadrant was significantly $(\sim 3$ times) longer in the exposed groups, and the time spent in the target quadrant was significantly $(\sim 2$ times) shorter (Figure 2). Exposed rats presented much lower numbers of correct annulus crossings than the controls.

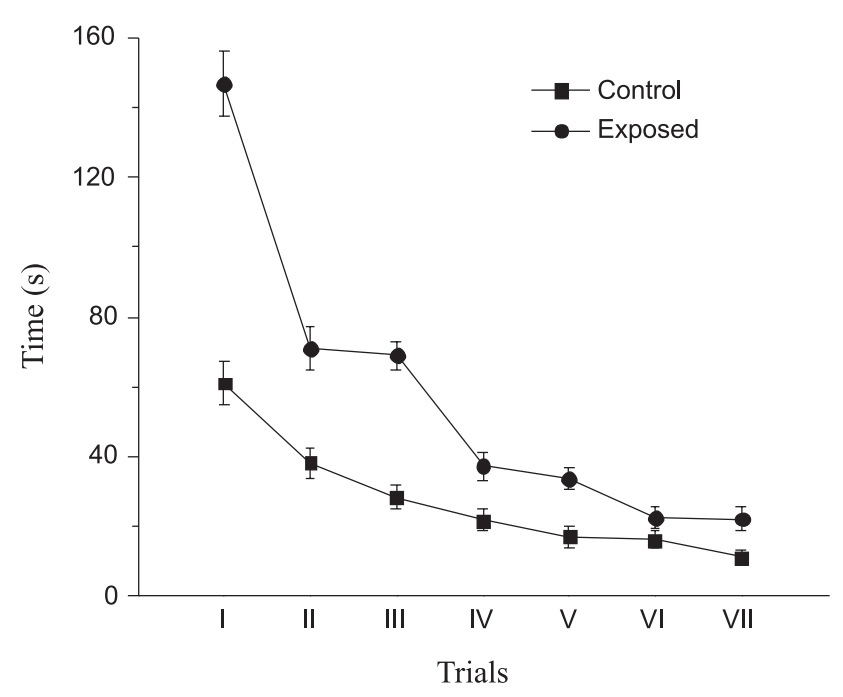

Figure 1 - Morris water maze escape latencies. Mean \pm STDEV for both groups of animas according to trial group. Both groups of animals improved their water maze acquisition performance i.e. decreased their latency to find the platform from the first to the last trial However, there was a notable difference in the latency times of the two groups during the training trails. 
A

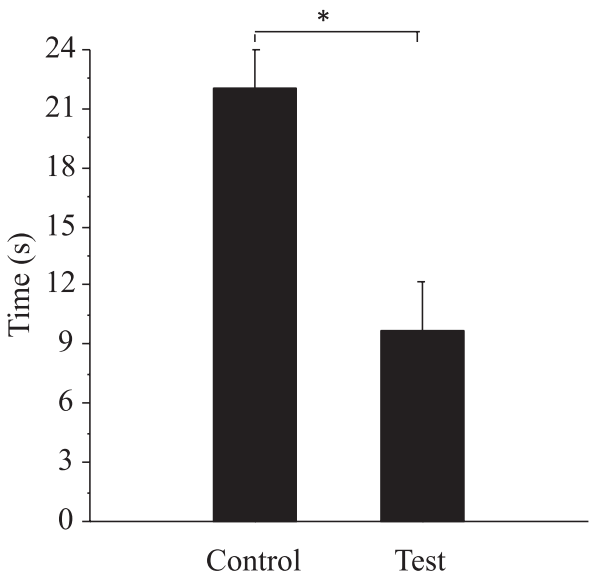

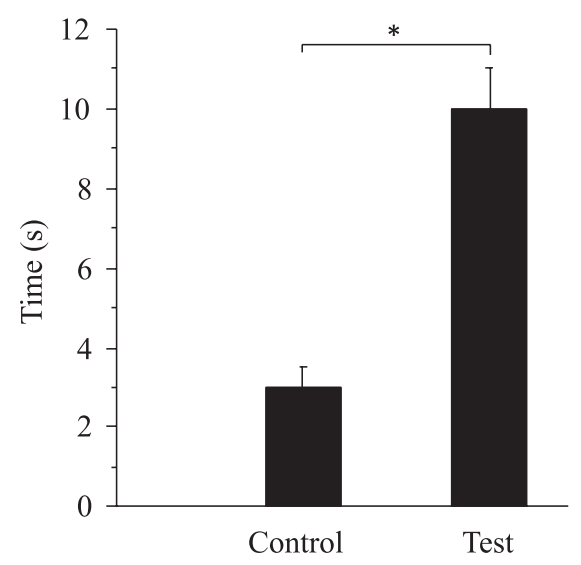

Figure 2 - Effect of mobile phone exposure on time spent in the target quadrant (A) and time taken to reach the target quadrant (B). Mobile phone animals spent less time in the target quadrant and needed more time to reach the target quadrant. Results are expressed as mean $\pm \mathrm{STDEV}$. $* \mathrm{p}<0.05$

\section{DISCUSSION}

Mobile phone exposure affected the acquisition of learned responses in the MWM test. The phone exposed animals exhibited longer latency than the control animals to reach the hidden platform in the trial sessions. In the retention test, their latency time to reach the target quadrant was longer and their time spent in the target quadrant was shorter. The phone-exposed animals were unable to recall the exact position of the hidden platform on the memory retention day, in spite of seven training sessions. This in turn points to the poor spatial navigation ability as well as the object-place configurations of the phone-exposed animals.

Cognitive function is known to be influenced by stress, both in animals and in humans. MWM learning is aversely motivated behavior, and, even when aversive factors are minimized to the extent possible, acquiring this task will always be a stressful event for the animals involved. Under these conditions, supplementary stress may revert profound effects on cognitive performance. The hippocampus plays a major role in spatial memory. Physiologically, cells in the hippocampus that display increased firing rates when the animal is in certain spatial locations are expected to be involved in spatial memory. ${ }^{3,4}$ The hippocampus encodes the spatial relationships between components of scenes or contexts and in the absence of this representation animals with hippocampal lesions will not be able to form the objectplace configurations that are important in episodic memory. ${ }^{5}$ Lesions in other brain regions like the striatum, basal forebrain, cerebellum and cerebral cortex were also shown to impair MWM performance in rats. Both neurons and glia interact dynamically to enable information processing and behavior. ${ }^{6}$ Mobile phone exposure induced glial reactivity ${ }^{7}$ in the rat brain probably due to the hypertrophy of glial cells and because prolonged exposure disrupted the integrity of the blood-brain barrier. ${ }^{8}$ The exposure of rats to mobile phone electromagnetic fields resulted in neuronal damage in the brain cortex, hippocampus, and basal ganglia ${ }^{9}$ and induced apoptosis in rat cortical primary neuronal cultures through a caspase-independent mitochondrial pathway that involves the AIF- apoptosis-inducing factor. ${ }^{10}$

Most of the cell phone safety recommendations are based on the basic sinusoidal wave, known as the carrier signal. However the danger does not come from this, but from the modulated signal that actually carries the data or voice information that operates at higher frequency. The cell receptors respond to this carrier signal, and get excited resulting in the impairment of delicate microtubular connections between the cells; in other words, the cells gets locked-up. Furthermore, this would lead to an increase in intracellular heavy metals and ROS generation. Reactive oxygen species (ROS) play a major role in electromagnetic radiation-induced tissue damage. Exposure to $900 \mathrm{MHz}$ electro magnetic radiation from a mobile phone has been shown to increase malondialdeyde, nitric oxide levels and activities of xanthine oxidase and glutathione peroxidase and to decrease the activity rates of superoxide dismutase and glutathione peroxidase activities in the rat brain. ${ }^{11}$ Exposure of adult Sprague-Dawley rats to regular cell phones resulted in mRNA up-regulation of several injury-associated proteins such as calcium ATPase, neural cell adhesion molecules, neural growth factor, and vascular endothelial growth factor. ${ }^{12}$ A study in guinea pigs has shown an increase in MDA and decrease in GSH levels and catalase activity in brain tissue after exposure to 890 to $915-\mathrm{MHz} \mathrm{RF}$ from a cellular phone. ${ }^{13}$ 
In conclusion, our preliminary results indicate that mobile phone exposure does induce behavioral changes in rats. The changes can be observed in terms of higher latency time to reach the target quadrant and less time spent in the target quadrant in the MWM test. This modification of rat behavior could either be due to microwave radiation from the phone or it could be due to vibration, or it may be linked to both. To determine which is more important further study in warranted.

\section{REFERENCES}

1. Schirmacher A, Winters S, Fischer S, Goeke J, Galla HJ, Kullnick U, et al. Electromagnetic fields $(1.8 \mathrm{GHz})$ increase the permeability to sucrose of the blood-brain barrier in vitro. Bioelectromagnetics. 2000; 21(5): $338-45$

2. Prato FS, Frappier JR, Shivers RR, Kavaliers M, Zabel P, Drost D,et al. Magnetic resonance imaging increases the blood-brain barrier permeability to 153 -gadolinium diethylenetriaminepentaacetic acid in rats. Brain Res. 1990; 523(2): 301-4.

3. Wisman LA, Sahin G, Maingay M, Leanza G, Kirik D. Functional convergence of dopaminergic and cholinergic input is critical for hippocampus-dependent working memory. J Neurosci. 2008; 28(31): 7797-807.

4. Talpos JC, Dias R, Bussey TJ, Saksida LM. Hippocampal lesions in rats impair learning and memory for locations on a touch-sensitive computer screen: the "ASAT" task. Behav Brain Res. 2008; 192(2): 216-25.

5. Lee I, Solivan F. The roles of the medial prefrontal cortex and hippocampus in a spatial paired-association task. Learn Mem. 2008; 15(5): 357-67.

6. Laming PR, Kimelberg H, Robinson S, Salm A, Hawrylak N, Müller $\mathrm{C}$, et al. Neuronal-glial interactions and behaviour. Neurosci Biobehav Rev. 2000; 24(3): 295-340.
7. Brillaud E, Piotrowski A, de Seze R. Effect of an acute $900 \mathrm{MHz}$ GSM exposure on glia in the rat brain: a time-dependent study. Toxicology. 2007; 238(1): 23-33.

8. Finnie JW, Blumbergs PC, Manavis J, Utteridge TD, Gebski V, Davies RA, et al. Effect of long-term mobile communication microwave exposure on vascular permeability in mouse brain. Pathology. 2004; 36(1): 96-7.

9. Salford LG, Brun AE, Eberhardt JL, Malmgren L, Persson BR. Nerve cell damage in mammalian brain after exposure to microwaves from GSM mobile phones. Environ Health Perspect. 2003; 111(7): 881-3

10. Joubert V, Bourthoumieu S, Leveque P, Yardin C. Apoptosis is induced by radiofrequency fields through the caspase-independent mitochondrial pathway in cortical neurons. Radiat Res. 2008; 169(1): 38-45

11. Ilhan A, Gurel A, Armutcu F, Kamisli S, Iraz M, Akyol O, et al. Ginkgo biloba prevents mobile phone-induced oxidative stress in rat brain. Clin Chim Acta. 2004; 340: 153-62.

12. Yan JG, Agresti M, Zhang LL, Yan Y, Matloub HS. Upregulation of specific mRNA levels in rat brain after cell phone exposure. Electromagn Biol Med. 2008; 27(2):147-54

13. Miyakoshi J, Takemasa K, Takashima Y, Ding GR, Hirose H, Koyama S. Effects of exposure to a $1950 \mathrm{MHz}$ radio frequency field on expression of Hsp70 and Hsp27 in human glioma cells. Bioelectromagnetics. 2005; 26(4): 251-7. 